\title{
Geometrical Representation of Sum Frequency Generation and Adiabatic Frequency Conversion
}

\author{
Haim Suchowski ${ }^{*}$ Dan Oron, and Yaron Silberberg \\ Department of Physics of Complex System, Weizmann Institute of Science, Rehovot, 76100, Israel \\ Ady Arie \\ School of Electrical Engineeing, Faculty of Engineering, Tel Aviv University, Tel Aviv, Israel
}

(Dated: October 24, 2018)

\begin{abstract}
We present a geometrical representation of sum frequency generation process in the undepleted pump approximation. The analogy of such dynamics with the known optical Bloch equations is discussed. We use this analogy to present a novel technique for the achievement of both high efficiency and large bandwidth in a sum frequency conversion processes using adiabatic inversion scheme, adapted from NMR and light-matter interaction. The adiabatic constraints are derived in this context. Last, this adiabatic frequency conversion scheme is realized experimentally by a proper design of adiabatic aperiodically poled KTP device, using quasi phased matched method. In the experiments we achieved high efficiency signal to idler conversion over a bandwidth of 140nm.

PACS numbers: $42.65 . \mathrm{Ky}, 42.25 . \mathrm{Fx}, 42.70 . \mathrm{Qs}$
\end{abstract}

The generation of tunable frequency optical radiation typically relies on nonlinear frequency conversion in crystals. In this process, light of two frequencies is mixed in a nonlinear crystal, resulting in the generation of a third color with their sum or difference frequency. These three-wave mixing processes, also known as frequency up-conversion or frequency down-conversion are typically very sensitive to the incoming frequencies, owing to lack of phase matching of the propagating waves. Thus, angle, temperature or other tuning mechanisms are needed to support efficient frequency conversion. This difficulty is of particular importance when trying to efficiently convert broadband optical signals, since simultaneous phase matching of a broad frequency range is difficult.

Solving the general form of the wave equations governing three wave mixing processes in nonlinear process is not an easy task. Under certain conditions these can be simplified to three nonlinear coupled equations. Further simplification can be applied when one incoming wave (termed pump) is much stronger than the other two. In the "undepleted pump" approximation, two linear coupled equation are obtained rather than three nonlinear ones [1. In the case of sum frequency generation (SFG) process, this simplified system possesses $\mathrm{SU}(2)$ symmetry, sharing its dynamical behavior with other two states systems, such as nuclear magnetic resonance (NMR) or the interaction of coherent light with a two-level atom. In this letter we explore the dynamical symmetry of SFG process in analogy with the well known two level system dynamics 2. We also apply a geometrical visualization using the approach presented by Bloch [3] and Feynman et al. 4] in NMR and light-matter interaction, respectively. The simple vector form of the coupling equation allows for new physical insight into the problem of fre-

*Electronic address: Haim.suchowski@weizmann.ac.il quency conversion, enabling a more intuitive understanding of the effects of spatially varying coupling and phase mismatch. The utility of this approach is demonstrated by introducing a robust, highly efficient broadband color conversion scheme, based on an equivalent mechanism for achieving full population inversion in atom-photon interactions known as Rapid Adiabatic Passage (RAP) scheme [5]. The demonstration is experimentally realized using an adiabatically varying aperiodically poled KTP (APPKTP) in a quasi phased matched (QPM) design.

Aperiodically poled structure have already been introduced for improving the bandwidth response of frequency conversion, but at a cost of a significantly reduced efficiency [6, 7, 8]. The broad bandwidth response is in particular importance for frequency conversion of ultrashort pulses. Chirped QPM gratings have been utilized to manipulate short pulses both in second harmonic generation (SHG) [9, 10, 11, difference frequency generation (DFG) 12, and in parametric amplification [10, 13. Recent application showed that by using a disordered material (random QPM), an extreme broad bandwidth was obtained, although at a price of severe reduction of the conversion efficiencies 14. While in standard frequency conversion processes, the crystal parameters and the pump intensity should be precisely matched in order to reach high conversion efficiency, here, by utilizing adiabatic frequency conversion one can still reach near to $100 \%$ conversion efficiency over a broad wavelength and temperature range. In our demonstration we succeed in achieving near complete conversion while maintaining extremely broad bandwidth of over 140nm.

Let us first consider the geometrical representation of SFG. In the undepleted pump approximation, the pump amplitude is assumed constant along the nonlinear crystal, and the following normalized coupled equations for 
a

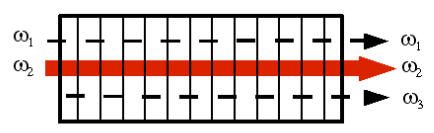

C

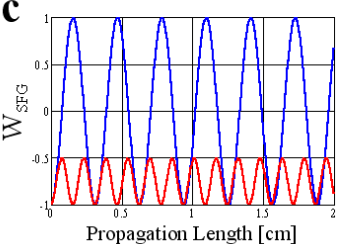

b

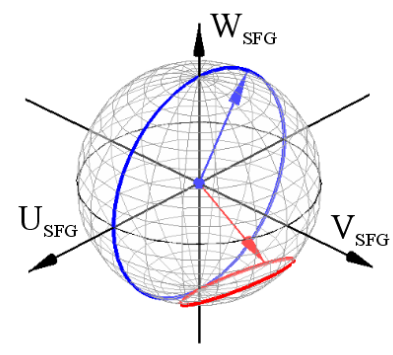

FIG. 1: Bloch sphere geometrical representation of SFG in the undepeleted pump approximation. The amplitude of $\omega_{2}$ (bold red arrow) is much larger than the amplitudes of $\omega_{1}, \omega_{3}$, and therefore remains constant along the propagation. The energy transfer between the incoming weak field $\left(\omega_{1}\right)$ and the converted SFG signal $\left(\omega_{3}\right)$ along the propagation is dictated by the coupling coefficient $\kappa$ and the phase mismatch $\Delta k$. a) A periodically poled quasi phased crystal designed to achieve a perfect phase matched interaction along the propagation. (b) Geometrical visualization of the SFG dynamics on a SFG Bloch Sphere. Two trajectories are plotted: perfect phase matching (blue, torque vector points to the equator) which can result in efficient conversion and a constant nonzero phase mismatch (red, torque vector points to a point in the south hemisphere), always resulting in an inefficient conversion process. (c) The projection of the trajectory onto the $\mathrm{z}$ axis yields the conversion efficiency.

the signal and idler can be constructed [1]:

$$
\begin{aligned}
& i \frac{d \tilde{A}_{1}}{d z}=\kappa \tilde{A}_{3} e^{-i \Delta k z} \\
& i \frac{d \tilde{A}_{3}}{d z}=\kappa^{*} \tilde{A}_{1} e^{+i \Delta k z}
\end{aligned}
$$

where $\Delta k=k_{1}+k_{2}-k_{3}$ is the phase mismatch, $\mathrm{z}$ is the position along the propagation axis, $\kappa$ is the coupling coefficient, and $\tilde{A}_{1}$ and $\tilde{A}_{3}$ are the normalized signal and idler amplitudes. The coupling coefficient and the normalized amplitudes are written below using the following quantities:

$$
\begin{aligned}
K_{1} & =\frac{16 \pi i \omega_{1}^{2} \chi^{(2)}}{k_{1} c^{2}} A_{2}^{*}, \quad K_{3}=\frac{16 \pi i \omega_{3}^{2} \chi^{(2)}}{k_{3} c^{2}} A_{2} \\
\kappa & =i \sqrt{K_{1} K_{3}} \\
\tilde{A}_{1} & =A_{1} / \sqrt{K_{1}}, \quad \tilde{A}_{3}=A_{3} / \sqrt{K_{3}}
\end{aligned}
$$

where $\omega_{1}$ and $\omega_{3}$ are the frequencies of the signal and idler, respectively, $k_{1}$ and $k_{3}$ are their associated wave numbers, $c$ is the speed of light in vacuum, $A_{1}, A_{2}, A_{3}$ are the signal, pump and idler amplitudes respectively, and $\chi^{(2)}$ is the $2^{\text {nd }}$ order susceptibility of the crystal (assumed to be frequency independent).

The dynamics of this system is dictated by the values of $\Delta k$ and $\kappa$. Full energy transfer from signal to idler (SFG process) or vice versa (DFG process) is usually achieved

by demanding perfect phase matching $(\Delta k(z)=0)$, and also by satisfying a constraint relating the propagation length in the crystal with the pump intensity, such that $\kappa \cdot z=(2 n+1) \pi$, where $\mathrm{n}$ is an integer. This is the only solution for a full conversion with a z-independent phase mismatch.

Equations 17 \& 1p have the same form as those describing the dynamics in two level systems. We adopt Feynman et al. 44 formulation and write the dynamics of this problem as a real three dimensional vector equation, which can be visualized geometrically on a sphere, known as Bloch sphere. In this context, we define a three dimensional state vector $\rho_{S F G}=(U, V, W)$ as follows:

$$
\begin{aligned}
U_{S F G} & =A_{3}^{*} A_{1}+A_{1}^{*} A_{3}, \\
V_{S F G} & =i\left(A_{3}^{*} A_{1}-A_{1}^{*} A_{3}\right), \\
W_{S F G} & =\left|A_{3}\right|^{2}-\left|A_{1}\right|^{2} .
\end{aligned}
$$

This vector represents the relation between the signal and idler along the crystal, and includes the coherence between the modes along the propagation. In particular, its z component $\left(W_{S F G}\right)$ gives information about the conversion efficiency. The south pole $\vec{\rho}=$ $(0,0,-1)$ corresponds to zero conversion $\left(A_{3}=0\right)$, while the north pole $\vec{\rho}=(0,0,1)$ corresponds to full conversion. In between, the conversion efficiency can be found as follow: $\eta=\left(W_{S F G}+1\right) / 2$. The torque vector $\vec{g}=(\operatorname{Re}\{\kappa\}, \operatorname{Im}\{\kappa\}, \Delta k)$ represents the coupling between these two modes, which contains also the phase mismatch parameter. In this form, the loss-free evolution equations can be written as a single vector precession equation:

$$
\frac{d \vec{\rho}_{S F G}}{d z}=\vec{g} \times \vec{\rho}_{S F G}
$$

The analogy suggests the equivalence between the quantities in the standard two-level system and those in the frequency-conversion problem: The population of the ground and excited states are analogous to the magnitude of the signal and idler fields, respectively. Time evolution is replaced by propagation in the longitudinal z-axis, and the detuning $\Delta$ is replaced by the phase-mismatch $\Delta k$ value; The analogy is further detailed in table I.

Presented in this context, the perfect phased matched solution for full conversion, as described above, has the same dynamical trajectory on the Bloch sphere surface as on-resonant light-matter interaction in atomic physics. This results in oscillatory dynamics between the two modes ("Rabi oscillations"). The case of an odd $\pi$-pulse is analogous to transferring the energy from $\omega_{1}$ (input signal) to the SFG/DFG output $\omega_{3}$, i.e. the SFG state vector is rotated from the south pole (north pole) to the north pole (south pole). Any phase mismatch will lead to a dynamics similar to a detuned case in light-matter interactions, which exhibit faster oscillations and lower conversion efficiencies, as shown in figure 1]

Due to the above, achievement of full energy transfer in an upconversion process is usually not robust, requiring several ingredients to be simultaneously satisfied. 
TABLE I: Analogy between Sum Frequency Generation (SFG) process in the undepleted pump approximation and the dynamics of a two level atomic system, induced by coherent light. The middle column describes the optical Bloch sphere realization of the two level systems (notation are taken from Allen \& Eberly [2]). The right column shows the analogous parameters of the SFG sphere realization.

\begin{tabular}{|c|c|c|}
\hline Parameter & Optical Bloch Sphere & SFG Sphere Realization \\
\hline Evolution parameter & time & $\mathrm{z}$ axis \\
Ground state population & $\left|a_{g}\right|^{2}$ & $\left|A_{1}\right|^{2}$ \\
Excited state population & $\left|a_{e}\right|^{2}$ & $\left|A_{3}\right|^{2}$ \\
Energy difference & $\omega_{0}=\omega_{f g}$ & $n\left(\omega_{2}\right) \omega_{2} / c$ \\
Detuning / Phase Mismatch & $\Delta$ & $\Delta k$ \\
"Rabi" frequency & $\Omega_{0}=\frac{1}{\hbar} \mu \cdot E_{i n}$ & $\kappa=\frac{4 \pi w_{1} w_{3}}{\left(k_{1} k_{3}\right)^{1 / 2} c^{2}} \chi^{(2)} \cdot E_{2}$ \\
Torque vector & $\Omega=\left(\operatorname{Re}\left\{\Omega_{0}\right\}, \operatorname{Im}\left\{\Omega_{0}\right\}, \Delta\right)$ & $g=(\operatorname{Re}\{\kappa\}, I m\{\kappa\}, \Delta k)$ \\
State vector - $\rho_{X}=\mathrm{U}$ & $a_{f}^{*} a_{g}+a_{g}^{*} a_{f}$ & $A_{3}^{*} A_{1}+A_{1}^{*} A_{3}$ \\
State vector $-\rho_{Y}=\mathrm{V}$ & $i\left(a_{f}^{*} a_{g}-a_{g}^{*} a_{f}\right)$ & $i\left(A_{3}^{*} A_{1}-A_{1}^{*} A_{3}\right)$ \\
State vector $-\rho_{Z}=\mathrm{W}$ & $\left|a_{f}\right|^{2}-\left|a_{g}\right|^{2}$ & $\left|A_{3}\right|^{2}-\left|A_{1}\right|^{2}$ \\
\hline
\end{tabular}

$\mathbf{a}$

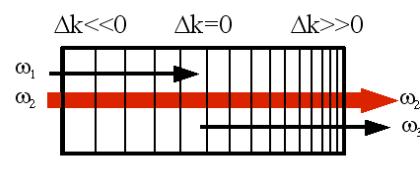

C

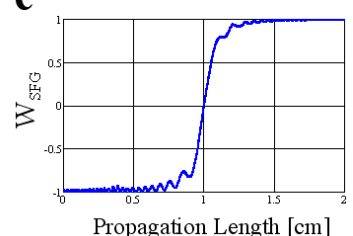

FIG. 2: Adiabatic conversion scheme of SFG in the undepleted pump approximation. a) Continuous adiabatic variation of the phase mismatch parameter is required. This can be achieved by slowly changing the poling periodicity along the propagation direction. b) The adiabatic following trajectory, where the torque vector initially points to the vicinity of the south pole, and ends up at the vicinity of the north pole. c) The projection of the trajectory onto the $z$ axis yields the conversion efficiency. In this trajectory, phase matching condition is fulfilled at $\mathrm{z}=1 \mathrm{~cm}$.

From the above analogy we can, however, adopt the RAP mechanism [5], where a strong chirped excitation pulse scans slowly through the resonance to achieve robust full inversion, to the realm of frequency conversion. Thus, in order to transfer from $A_{1}(z)$ to $A_{3}(z)$ for a broadband of wavelengths, the phase mismatch parameter, $\Delta k(z)$ has to change adiabatically from a big negative value to a big positive value (or vice versa). The adiabaticity conditions require that:

$$
\begin{aligned}
|\Delta k| & > \\
\Delta k(z=0)<0 & , \quad \Delta k(z=L)>0 \\
\left|\frac{d \Delta k}{d z}\right| & <\frac{\left(\Delta k^{2}+\kappa^{2}\right)^{3 / 2}}{\kappa}
\end{aligned}
$$

The first two conditions deal with the maximal magnitude of the phase mismatch, which should be very large in comparison to the value of $\kappa$, and the interaction has to start with a large negative (or positive) value of phase mismatch, and end with a large positive (or negative) value. The third condition is the most acute one, dealing with the rate of change of the phase mismatch during propagation. To satisfy this condition, the phase mismatch has to be vary slowly as compared to the square of the coupling term in this nonlinear process. If the rate of variation is not slow enough (left hand side value of inequality 5 ; is not small enough), or the coupling coefficient is not large enough, this inequality won't be satisfied and the conversion efficiency will be poor. Figure 2 demonstrate the case when all those constraints are satisfied and full frequency conversion is achieved.

In the experimental realization of this adiabatic conversion scheme we choose to utilize the QPM technique 15. By tuning the spatial structure of the domains, this technique allows not only to achieve phase mismatch that is close to zero along the propagation axis, but almost any desired function of the phase mismatched parameter. By proper design of the periodicity of the poling, one can achieve an effective phase mismatch parameter which is the summation of the process phase mismatch and the artificial phase-mismatch $\Delta k_{\Lambda}(z)$ :

$$
\Delta k_{\text {eff }}(z)=k_{\text {signal }}+k_{\text {pump }}-k_{\text {idler }}-\Delta k_{\Lambda}(z) .
$$

The desired value of the phase-mismatch parameter is achieved by poling the QPM structure using the approximate relation: $\Delta k_{\Lambda}(z)=\frac{2 \pi}{\Lambda(z)}$, where $\Lambda(z)$ is the local poling period.

We demonstrated our idea by an adiabatic APPKTP which was designed to satisfy the constraints posed by equations 5. The periodicity was varied from $14.6 \mu \mathrm{m}$ to $16.2 \mu \mathrm{m}$ along a crystal length of $L=20 \mathrm{~mm}$, to induce a linear adiabatic change in $k_{e f f}(z)$. The design was carried out by a numerical simulations of the process propagation using the finite difference method. We assumed plain wave approximation and took $\chi^{(2)}=32 \frac{\mathrm{pm}}{\mathrm{V}}$. We used an OPO (Ekspla NT342) as our sources which produced a strong pump at $1064 \mathrm{~nm}(6 \mathrm{~ns}, 130 \mu \mathrm{J})$, and 


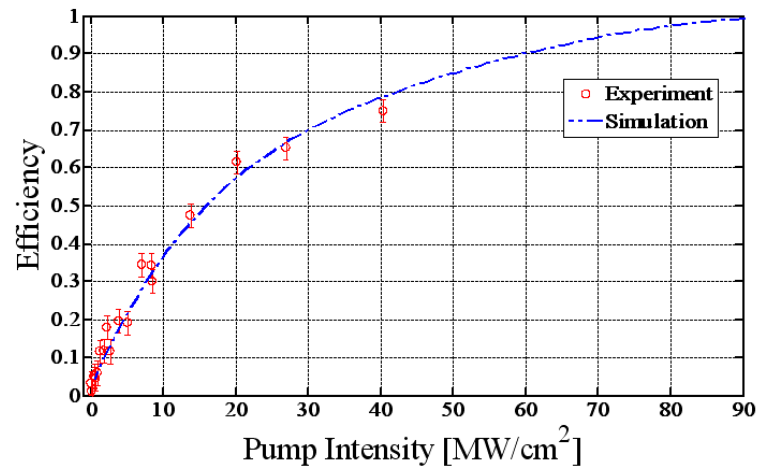

FIG. 3: Conversion efficiency as a function of pump intensity with a signal wavelength of $1530 \mathrm{~nm}$ at room temperature $\left(21^{\circ} \mathrm{C}\right)$. A good correspondence between the experimental results (shown in red) and the simulation of the design (dasheddotted blue). The maximal conversion efficiency achieved was $74 \% \pm 3 \%$.

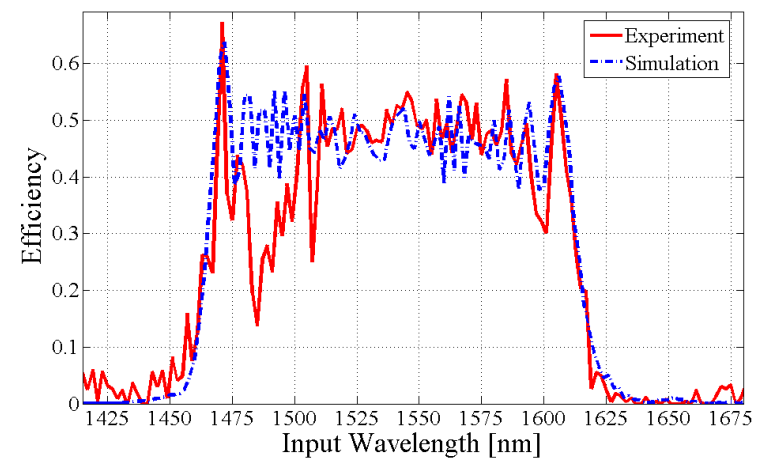

FIG. 4: Conversion efficiency as a function of input wavelength using the adiabatic APPKTP design at a pump intensity of $15 \mathrm{MW} / \mathrm{cm}^{2}$. A good correspondence between the experimental results (shown in solid red) and the simulation of the design (dashed-dotted blue) is shown. The low efficiency around $1485 \mathrm{~nm}$ is associated with a manufacturing defect.

a tunable signal varied from $1400 \mathrm{~nm}$ to $1700 \mathrm{~nm}(5 \mathrm{~ns}$, $1 \mu J)$. The pump and the signal, both polarized in the extra-ordinary axis were spatially overlapped and focused collinearly into the crystal having waists of $150 \mu \mathrm{m}^{2}$ and $120 \mu \mathrm{m}^{2}$, respectively. These values guarantee that the Rayleigh range is larger than the crystal length and thus the plane wave approximation of our simulations holds. We collected both the input wavelength (signal) and output SFG wavelength (idler) after their propagation in the crystal and recorded by an InGaAs detector, and a cooled CCD spectrometer, respectively.

Our demonstration consists of two sets of experiments. First, to demonstrate the crucial requirement of the strong pump, we examined the dependence of the conversion efficiency on the pump intensity. In the second experiment we measure the conversion efficiency as a function of signal wavelength at a constant pump intensity.
In both cases, the conversion efficiency was measured by comparing the signal intensity with and without the presence of the pump. This was checked to be completely correlated with the observed SFG intensity and free of thermal effects.

In Fig. 3 we plot the conversion efficiency as a function of the pump peak intensity for a fixed signal frequency $1530 \mathrm{~nm}$. A very good correspondence is obtained with the simulation. The maximal efficiency which was achieved with our maximal pump intensity was $74 \% \pm 3 \%$.

In a second experiment, when the input wavelength was varied, we chose to work at a moderate pump intensity of $15 \mathrm{MW} / \mathrm{cm}^{2}$ and at room temperature. We show in Figure 4 efficient broadband conversion of over $140 \mathrm{~nm}$ wide (1470nm to $1610 \mathrm{~nm})$. This is in a good correspondence with the numerical simulation of the design (dashed-dotted blue), except for a small region of low efficiency around $1485 \mathrm{~nm}$, which is associated with a manufacturing defect, thus causing the violation of the adiabaticity condition. For comparison, a conventional efficient converter (a periodically poled structure designed to achieve perfect phase matching), would lead to efficient broadband conversion only for $2 \mathrm{~nm}$ bandwidth.

Other experiments showing the robustness of the adiabatic conversion scheme to errors in incidence angle, crystal temperature and pump frequency (as compared to the periodically poled design), and exhibiting color tunability of the conversion band by temperature control will be presented in a forthcoming publication.

In conclusion, we show that the use of a geometrical Bloch sphere visualization to describe evolution of the complex mode amplitudes, gives information on their relative phases as well as intensities. We demonstrated high efficiency wavelength conversion for a wide range of frequencies, by using the adiabatic conversion method adapted from other well known two level systems. It is important to note that quasi phase matched crystal is only one possible realization; the same mechanism can be applied, for example, by inducing a temperature gradient on a perfect phased matched crystal, or by any other mechanism fulfilling the adiabatic constraint of Eq. 5. The present scheme can be utilized for efficient frequency conversion of broadband signals as well as ultrashort pulses. This analysis holds promise for ultrashort pulse conversion in a wide range of frequencies from UV to far IR. It may be particularly useful in the efficient upconversion of weak infrared signals to the nearinfrared or visible range, used in atmospherical observation [16, 17. The generalization of this analogy to higher order nonlinear processes interaction can be made, and known schemes from atom-photon interaction or NMR, such as Stimulated Raman Adiabatic Passage (STIRAP) [18] can be adopted.

This research was supported by Israel Science Foundation (Grants No. 1621/07 and 960/05). H. S. is grateful to the Azrieli Foundation for the award of an Azrieli Fellowship. 
[1] R. W. Boyd Nonlinear Optics, Academic Press, (2005).

[2] L. D. Allen and J. H. Eberly Optical Resonance and Two Level Atoms, Wiley, New York, (1975).

[3] F. Bloch, Phys. Rev. 70, 460 (1946).

[4] R. Feynman, F. Venon, and R. Hellwarth, J. of App. Phys. 28, 49 (1957).

[5] A. Massiach Quantum Mechanics, Vol. II, NorthHolland, (1961).

[6] M. L. Bortz, M. Fujimura, and M. Fejer, Elec. Lett. 30, 34 (1994).

[7] K. Mizuuchi, K. Yamamoto, M. Kato, and H. Sato, IEEE J. of Quant. Elec. 30, 1596 (1994).

[8] H. Guo, S. H. Tang, Y. Qin, and Y. Y. Zhu, Phys. Rev. E. 71 (2005).

[9] M. Arbore, A. Galvanauskas, D. Harter, and M. Chou, M.H. Fejer, Opt. Lett. 22, 1341 (1997).

[10] G. Imeshev, M. Arbore, M. Fejer, A. Galvanauskas, M. Fermann, and D. Harter, J. Opt. Soc. Am. B 17,
$304(2000)$.

[11] D. S. Hum and M. M. Fejer, C. R. Physique 8, 180 (2007).

[12] G. Imeshev, M. Fejer, A. Galvanauskas, and D. Harter, Opt. Lett. 26, 568 (2001).

[13] M. Charbonneau-Lefort, B. Afeyan, and M. M. Fejer, J. Opt. Soc. Am. B 25, 463 (2008).

[14] Baudrier-Raybaut, R. Haidar, P. Kupecek, P. Lemasson, and E. Rosencher, Nature 432, 374 (2004).

[15] J. Armstrong, N. Bloembergen, J. Ducuing, and P. Pershan, Phys. Rev. 127, 1918 (1962).

[16] S. Brustlein, L. Del Rio, A. Tonello, L. Delage, F. Reynaud, H. Herrmann, and W. Sohler, Phy. Rev. Lett. 100, 153903 (2008).

[17] R. W. Boyd, Opt. Eng. 16, 563 (1977).

[18] N. V. Vitanov, T. Halfmann, B. W. Shore, and K. Bergmann, Ann. Rev. of Phys. Chem. 52, 763 (2001). 